\title{
Optogenetic control of Neisseria meningitidis Cas9 genome editing using an engineered, light-switchable anti-CRISPR protein
}

\author{
Mareike D. Hoffmann ${ }^{1, \dagger}$, Jan Mathony ${ }^{2,3,4, \dagger}$, Julius Upmeier zu Belzen ${ }^{5}$, Zander Harteveld ${ }^{6,7}$, \\ Sabine Aschenbrenner ${ }^{2,3}$, Christina Stengl ${ }^{8}$, Dirk Grimm ${ }^{1,9,10}$, Bruno E. Correia ${ }^{6,7}$, \\ Roland Eils ${ }^{5,11, *}$ and Dominik Niopek ${ }^{\circledR 2,3, *}$
}

\begin{abstract}
${ }^{1}$ Dept. of Infectious Diseases/Virology, Medical Faculty, University of Heidelberg, 69120 Heidelberg, Germany, ${ }^{2}$ Centre for Synthetic Biology, Technical University of Darmstadt, 64287 Darmstadt, Germany, ${ }^{3}$ Department of Biology, Technical University of Darmstadt,64287 Darmstadt, Germany, ${ }^{4} \mathrm{PhD}$ Student, BZH graduate school, Heidelberg University, 69120 Heidelberg, Germany, ${ }^{5}$ Digital Health Center, Berlin Institute of Health (BIH) and Charité, Berlin 10178, Germany, ${ }^{6}$ Institute of Bioengineering, École Polytechnique Fédérale de Lausanne, Lausanne $\mathrm{CH}-1015$, Switzerland, ${ }^{7}$ Swiss Institute of Bioinformatics (SIB), Lausanne $\mathrm{CH}-1015$, Switzerland, ${ }^{8}$ BioQuant, University of Heidelberg, 69120 Heidelberg, Germany, ${ }^{9}$ BioQuant, Cluster of Excellence CellNetworks, University of Heidelberg, 69120 Heidelberg, Germany, ${ }^{10}$ German Center for Infection Research (DZIF) and German Center for Cardiovascular Research (DZHK), partner site Heidelberg, 69120 Heidelberg, Germany and ${ }^{11}$ Health Data Science Unit, BioQuant and Medical Faculty of Heidelberg University, Heidelberg 69120, Germany
\end{abstract}

Received November 27, 2019; Revised October 30, 2020; Editorial Decision November 20, 2020; Accepted November 30, 2020

\begin{abstract}
Optogenetic control of CRISPR-Cas9 systems has significantly improved our ability to perform genome perturbations in living cells with high precision in time and space. As new Cas orthologues with advantageous properties are rapidly being discovered and engineered, the need for straightforward strategies to control their activity via exogenous stimuli persists. The Cas 9 from Neisseria meningitidis ( $\mathrm{Nme}$ ) is a particularly small and target-specific Cas 9 orthologue, and thus of high interest for in vivo genome editing applications. Here, we report the first optogenetic tool to control NmeCas9 activity in mammalian cells via an engineered, light-dependent anti-CRISPR (Acr) protein. Building on our previous Acr engineering work, we created hybrids between the NmeCas9 inhibitor AcrllC3 and the LOV2 blue light sensory domain from Avena sativa. Two AcrllC3-LOV2 hybrids from our collection potently blocked NmeCas9 activity in the dark, while permitting robust genome editing at various endogenous loci upon blue light irradiation. Structural analysis revealed that, within these hybrids, the LOV2 domain is located in striking proximity to the Cas 9 binding surface. Together, our
\end{abstract}

work demonstrates optogenetic regulation of a type II-C CRISPR effector and might suggest a new route for the design of optogenetic Acrs.

\section{INTRODUCTION}

Genome engineering technologies based on CRISPR (clustered regularly-interspaced short palindromic repeats)-Cas systems facilitate site-specific targeting and manipulation of genes in living cells (1-3) and currently transform many areas of biomedical research. CRISPR systems can generally be subdivided into two classes. Class 1 systems comprise multi-protein effectors, while class 2 systems employ single protein effectors (4), which mediate targeted cleavage of nucleic acids. The class 2 type-II effectors, typically applied for genome engineering, comprise a Cas9 nuclease as the protein component and a single guide RNA (sg)RNA, which directs the Cas9 nuclease to selected nucleic acid targets by means of sequence complementarity. Due to their simplicity and versatility, class 2 CRISPR systems enable a plethora of applications including targeted induction of DNA doublestrand breaks for genome editing $(1,2,5)$, regulation of endogenous transcription $(5,6)$, epigenetic reprogramming (79), DNA labeling $(10,11)$ and base editing $(12,13)$.

The type II-A CRISPR-Cas9 from Streptococcus pyogenes (SpyCas9) is the most widely applied CRISPR-Cas9 orthologue. Due to its large size of 1,368 amino acids

\footnotetext{
${ }^{*}$ To whom correspondence should be addressed. Tel: +49 615116 21941; Fax: +49 615116 21942; Email: dominik.niopek@bioquant.uni-heidelberg.de Correspondence may also be addressed to Roland Eils. Tel: +49 3045054 3084; Fax: +49 304507576902 ; Email: roland.eils@bihealth.de

${ }^{\dagger}$ The authors wish it to be known that, in their opinion, the first two authors should be regarded as Joint First Authors.
} 
$(158 \mathrm{kDa})$ that hampers delivery by viral vectors and its high off-target rates (14-16), however, alternative CRISPRCas9 orthologues gained attention. A particularly interesting candidate is the type II-C Cas9 from Neisseria meningitidis (NmeCas9). With only 1081 amino acids (124 kDa), NmeCas9 is considerably smaller than SpyCas9. On top, NmeCas9 exhibits an exceptionally high target specificity, possibly due its longer target recognition sequence of about 24 nucleotides and also a longer PAM sequence $\left(\mathrm{N}_{4}\right.$ GATT for NmeCas9 versus NGG for SpyCas9) $(17,18)$. These properties render $N m e$ Cas 9 a powerful tool for various applications, including in vivo gene editing (19) and also RNA-induced genome binding via catalytically impaired NmeCas9 mutants $(20,21)$.

The ability to control and fine-tune NmeCas9 activity via exogenous stimuli would further enhance the precision at which CRISPR genome perturbations can be made. Unlike SpyCas9, for which a whole battery of tools exist that facilitate its conditional activation by chemical triggers (22-25), light (26-30) or temperature $(31,32)$, no method for conditional activation of NmeCas 9 by exogenous triggers has yet been reported.

Anti-CRISPR (Acr) proteins are bacteriophage-derived antagonists of CRISPR-Cas systems (21,33-44). They represent a highly diverse class of proteins practically without structural and sequence homology to other proteins $(39,40)$. Acrs inhibit Cas nucleases by various mechanisms, including inhibition of DNA binding (45-48), cleavage of sgRNAs (49), masking catalytic residues and/or inducing Cas9 dimerization (50). AcrIIC3 is a remarkably potent NmeCas9 inhibitor initially discovered in a putative prophage element within the Neisseria meningitidis genome (40). It binds the catalytic HNH domain and induces dimerization of $N m e$ Cas9 via the REC lobe in a stoichiometric ratio of AcrIIC3:Cas9 = 2:2, thereby blocking DNA binding $(50,51)$.

Here, we report the engineering and application of CASANOVA-C3 (for CRISPR-Cas9 activity switching via a novel optogenetic variant of AcrIIC3), a lightdependent anti-CRISPR protein for conditional activation of NmeCas9. Building on our recent AcrIIA4 engineering work $(30,52)$, we created hybrids between AcrIIC3 and the Avena sativa (As)LOV2 photosensory domain by systematically sampling AcrIIC3 surface sites. Following screening and optimization, two AcrIIC3-LOV2 hybrid variants were obtained, which potently inhibit NmeCas9 in the dark, while releasing its activity upon blue light irradiation (Figure 1A). We demonstrate light-dependent editing of various genomic loci upon transient transfection and Adenoassociated virus (AAV)-mediated transduction. Finally, using structural modeling, we show that our CASANOVA-C3 design represents an unconventional, yet potentially powerful and versatile blueprint to engineer light-dependent protein-protein interactions.

\section{MATERIALS AND METHODS}

\section{General methods and cloning}

A list of all constructs created and used in this study is provided in Supplementary Table S1. Annotated plasmid sequences (SnapGene DNA files) are provided as Supplementary Data. Oligonucleotides and double-stranded DNA fragments were obtained from Integrated DNA Technologies. AAV plasmids were generated via restriction enzyme cloning, all other constructs were cloned by Golden Gate assembly (53). The vector for co-expression of NmeCas9 and the VEGFA sgRNA was previously published by us (54). All other sgRNAs were cloned into plasmid pEJS654 All-in-One AAV-sgRNA-hNmeCas9 (kind gift from Erik Sontheimer, Addgene plasmid \#112139) via the SapI restriction sites. A list of genomic target sites is provided in Supplementary Table S2. The dual luciferase reporter was previously reported by us (52). AcrIIC3-LOV2 hybrid constructs were created by inserting the LOV2 domain into our published CMV promoter-driven AcrIIC3 expression vector (Addgene plasmid \#120301) (54). To this end, the AcrIIC3 vector was linearized by an around-the-horn PCR using primers carrying BbsI restriction sites as $5^{\prime}$ extension. The LOV2 domain was PCR-amplified from the vector CMV-CASANOVA (Addgene plasmid \#113035) previously reported by us (30). LOV2 primer $5^{\prime}$ extensions contained BbsI sites compatible with the vector amplicon BbsI sites and - optionally - sequences encoding flexible, glycineserine linkers. Golden Gate cloning was then used to assemble AcrIIC3-LOV2 hybrids. AcrIIC3-LOV2 AAV vectors were created by replacing the wild-type AcrIIC3 coding sequence in vector AAV CMV-driven AcrIIC3-scaffold (2xBsmBI sites) previously reported by us (54) by the AcrIIC3LOV2 coding sequences via the XhoI and NheI restriction sites. The HA-tagged AcrIIC3 plasmids were generated by introducing the 3xHA-tag as annealed oligos at the $\mathrm{N}$ terminus of the Acr by Golden Gate cloning.

All PCRs were performed using the Q5 Hot Start HighFidelity Polymerase (New England Biolabs, NEB). PCR products were analysed on 1-2\% TAE or TBE agarose gels. The desired bands were cut out and extracted from the gel using a QIAquick Gel Extraction Kit (Qiagen). Restriction digests and Golden Gate assembly were performed according to the manufacturer's protocols with enzymes obtained from Thermo Fisher Scientific or NEB. Fragments were ligated using T4 DNA Ligase (Thermo Fisher Scientific) and constructs transformed into chemically-competent Top10 cells (Thermo Fisher Scientific). Plasmid DNA was purified using QIAprep Spin Miniprep, Plasmid Plus Midi or Plasmid Maxi Kit (all Qiagen).

\section{Cell culture and AAV production}

HEK293T (human embryonic kidney) cells were maintained in DMEM (Thermo Fisher Scientific) supplemented with $10 \%(\mathrm{v} / \mathrm{v})$ fetal calf serum (FCS, Biochrom AG), 2 $\mathrm{mM}$ L-glutamine (Thermo Fisher Scientific) and $100 \mathrm{U} / \mathrm{ml}$ penicillin and $100 \mu \mathrm{g} / \mathrm{ml}$ streptomycin (Thermo Fisher Scientific). The medium for Huh7 cells was additionally supplemented with $1 \mathrm{mM}$ non-essential amino acids (Thermo Fisher Scientific). Cells were cultured at $37^{\circ} \mathrm{C}$ and $5 \% \mathrm{CO}_{2}$ in a humidified incubator and passaged every 2-3 days (i.e. when about $90 \%$ confluent). Cells were authenticated and tested for mycoplasma contamination prior to use via a commercial service (Multiplexion). 
To produce AAV lysates, low-passage HEK293T cells were seeded at a density of 350000 cells per well into six-well plates (CytoOne) using $4 \mathrm{ml}$ of medium per well. The next day, cells were triple-transfected with (i) AAV vector (transgene flanked by AAV inverted terminal repeats (ITRs)), (ii) the AAV helper plasmid carrying the rep and cap genes of AAV serotype 2 and (iii) an adenoviral plasmid providing helper functions for AAV production using $8 \mu 1$ TurboFect Transfection Reagent per well (Thermo Fisher Scientific). Seventy-two hours post-transfection, cells were flushed off the culture plate surface by pipetting and collected into the medium. Samples were then spun down, the supernatant (medium) was discarded and the cell pellet was resuspended in $300 \mu 1$ PBS. Cells were then lysed by applying five freezethaw cycles of snap-freezing in liquid nitrogen, followed by incubation at $37^{\circ} \mathrm{C}$ in a water bath. Subsequently, the cell lysate was centrifuged at $18000 \mathrm{~g}$ at $4^{\circ} \mathrm{C}$ for $10 \mathrm{~min}$ to remove cell debris and the AAV-containing supernatant was kept at $4{ }^{\circ} \mathrm{C}$ until use. Lysates were stored for no longer than 3 weeks.

\section{Blue light setup}

Blue light exposure of the samples was achieved with a custom-made LED setup, consisting of six blue light high power LEDs (type CREE XP-E D5-15; emission peak $\sim 460 \mathrm{~nm}$; emission angle $\sim 130^{\circ}$; LED-TECH.DE) connected to a Switching Mode Power Supply (Manson; HCS3102). The setup was controlled by a custom Python script, running on a Raspberry Pi. LEDs were positioned underneath a transparent table made of acrylic glass and positioned inside a cell culture incubator. Culture plates with samples were positioned on top of the table, i.e. they were irradiated from below through the acrylic glass and culture plate's transparent bottom. Illumination intensity was set to $3 \mathrm{~W} / \mathrm{m}^{2}$ and regularly confirmed by measurements with a LI-COR LI-250A light meter. Pulsatile illumination was used ( $5 \mathrm{~s}$ on, $10 \mathrm{~s}$ off). Dark control samples were kept in the same incubator, but protected from light by covering the transparent sample plate parts with black vinyl foil (Starlab). A detailed description of the blue light setup has been previously reported by us ((55); 'CASANOVA Step by Step: A protocol for optogenetic control of CRISPR/Cas9 with an engineered, light-dependent anti-CRISPR protein' (30)).

\section{Luciferase assay}

12500 cells/well were seeded into black, clear-bottom 96well plates (Corning). The next day, cells were transfected using Lipofectamine 3000 Reagent (Thermo Fisher Scientific), following the manufacturer's protocol. $200 \mathrm{ng}$ of total DNA per well comprising equal amounts (plasmid mass) of (i) dual luciferase reporter plasmid, (ii) $\mathrm{NmeCas} 9$ and sgRNA encoding plasmid and (iii) Acr-LOV2 constructs were co-transfected. For the reporter-only and no-Acr controls, the DNA was topped up to $200 \mathrm{ng}$ with stuffer DNA. The reporter construct encoded a Renilla and firefly luciferase as well as a sgRNA targeting a sequence stretch implanted in frame with $\left(5^{\prime}\right.$ of $)$ the firefly luciferase coding sequence. Forty-eight hours post-transfection, cells were washed with $1 \times$ PBS (Sigma-Aldrich) and lysed in Passive
Lysis Buffer (Promega). Subsequently, the luciferase activities were measured with the Dual-Glo Luciferase Assay System (Promega) on a GLOMAX discover or GLOMAX 96microplate luminometer (both Promega). Integration times of $10 \mathrm{~s}$ were used; delay between automated substrate injection and measurement was $2 \mathrm{~s}$. Firefly luciferase photon counts were normalized to Renilla luciferase photon counts. Finally, obtained values were normalized to the reporter only controls in the light or dark, yielding the reported normalized luciferase activity.

\section{MTT assay}

12500 HEK293T cells per well were seeded into black, clear-bottom 96-well plates (Corning). The next day, cells were transfected as for the luciferase assays (see above). Post-transfection, cells were either kept in the dark or irradiated with pulsed blue light. After $48 \mathrm{~h}$, illumination was stopped and non-transfected cells were treated with $0.001 \%$, $0.01 \%, 0.1 \%, 1 \%$ or $10 \%$ of Triton. Immediately thereafter, the MTT assay was performed using the MTT Cell Proliferation Assay Kit (Cayman Chemical) and by following the manufacturer's instructions.

\section{T7 endonuclease I assay (T7 assay)}

HEK293T cells were seeded into black, clear-bottom 96well plates using 12500 cells and $100 \mu 1$ medium per well (Corning). The next day, cells were transfected with $150 \mathrm{ng}$ total DNA per well using the Lipofectamine 3000 reagent and following the manufacturer's protocol. The vector mass ratios of Cas9/sgRNA and AcrIIC3-LOV2 construct used during transfection are indicated in the corresponding figures. In case of the Acr titration (Figure 2C), the cells were transfected using the JetPrime transfection reagent (Polyplus) according to the manufacturer's protocol and $150 \mathrm{ng}$ DNA per well. To keep the total amount of DNA constant across all samples and controls, the DNA was topped up to $150 \mathrm{ng}$ with stuffer DNA if required. The plasmid ratios are indicated at the respective figure panels.

For AAV experiments, 3500 HEK293T cells or 3000 Huh7 cells were seeded per well into black, clear-bottom 96well plates (Corning). The cells were co-transduced twice, i.e. on two consecutive days, with $80 \mu \mathrm{AAV}$ lysate. The lysate comprised Cas9/sgRNA and AcrIIC3-LOV2 AAV lysate in a volumetric ratio as indicated in the corresponding figures. For the controls without Acr, Cas9/sgRNA AAV lysate was topped up to $80 \mu 1$ with PBS to keep the transduction volume constant between all samples.

Cells were lysed $72 \mathrm{~h}$ post-transfection or post (first) transduction using the DirectPCR lysis reagent (PeqLab) supplemented with $200 \mu \mathrm{g} / \mathrm{ml}$ proteinase K (Roche Diagnostics). The targeted genomic locus was then PCRamplified with primers flanking the expected cutting site (Supplementary Table 3) using Q5 Hot Start High-Fidelity Polymerase (NEB). Five $\mu$ lof the resulting amplicon were diluted $1: 4$ in $1 \times$ buffer 2 (NEB), followed by denaturation and re-annealing in a nexus GSX1 Mastercycler (Eppendorf) by running the following protocol: Denaturation: $95^{\circ} \mathrm{C}$ for $5 \mathrm{~min}$; re-annealing: cooling down to $85^{\circ} \mathrm{C}$ at a ramp rate of $0.2^{\circ} \mathrm{C} / \mathrm{s}$ followed by cooling down to $25^{\circ} \mathrm{C}$ 
at a ramp rate of $0.1^{\circ} \mathrm{C} / \mathrm{s}$. Next, $0.5 \mu \mathrm{T} \mathrm{T} 7$ endonuclease I (NEB) was added and the samples were incubated at $37^{\circ} \mathrm{C}$ for $15 \mathrm{~min}$. Samples were then analysed on $2 \%$ Tris-borateEDTA agarose gels. Gel documentation was performed using a Gel iX20 system equipped with a 2.8 megapixel/14 bit scientific grade CCD camera (INTAS). Intensities of DNA fragments were quantified using the ImageJ gel analysis tool (http://imagej.nih.gov/ij/). Finally, indel percentages were calculated using the following formula: indel $(\%)=100 \times$ $(1-(1-$ fraction cleaved $) 1 / 2)$, whereas the fraction cleaved $=\sum($ Cleavage product bands $) / \sum($ Cleavage product bands + PCR input band). Full-length gel images are shown in Supplementary Figure S1.

\section{TIDE sequencing}

Cells were lysed and genomic target loci were PCRamplified as described for the T7 assay. PCR amplicons were then resolved by gel electrophoresis followed by DNA isolation with a QIAquick Gel Extraction Kit (Qiagen). The amplicons were Sanger sequenced (Eurofins Genomics) and sequencing chromatograms were analysed using the TIDE web tool to determine the percentage of indels (https://tide. deskgen.com/) (56).

\section{NGS sequencing}

Cells were lysed in DirectPCR lysis reagent (PeqLab) supplemented with $200 \mu \mathrm{g} / \mathrm{ml}$ proteinase K (Roche Diagnostics) and the edited loci were amplified by PCR. The PCR primers carried multiplexing barcodes and the partial Illumina adapters as overhangs. The resulting amplicons were analyzed by gel electrophoresis and subsequently DNA was isolated with a QIAquick Gel Extraction Kit (Qiagen). The DNA concentration was adjusted to $20 \mathrm{ng} / \mu \mathrm{l}$ and up to six barcoded samples were pooled. Amplicon sequencing was performed by the Genewiz Amplicon-EZ service. The data was analysed using the Sabre (https://github.com/najoshi/ sabre) and CRISPresso 2.0 (https://github.com/pinellolab/ CRISPResso2 (57)) packages in order to determine the indel frequencies.

\section{Western blot}

HEK293T cells were seeded into six-well plates (CytoOne) at $2.5 \times 10^{5}$ cells/well and transfected with $2 \mu \mathrm{g}$ total DNA, comprising plasmids encoding (i) NmeCas9 with a nontargeting sgRNA and (ii) the respective Acr in a mass ratio of 1:1. Transient transfection was performed using the JetPrime reagent according to the manufacturer's protocol. At the indicated time points, the media was aspirated, the cells were washed with PBS and $150 \mu$ l of protein lysis buffer (150 mM NaCl, $10 \mathrm{mM}$ Tris, $1 \mathrm{mM}$ EDTA, 0.5\% NP-40 and $10 \%$ cOmplete Protease Inhibitor (Roche), $\mathrm{pH} 8.0$ ) was added. After scraping the cells off the culture plate surface, remaining debris was pelleted by centrifugation at $10000 \mathrm{~g}$ for $5 \mathrm{~min}$ at $4^{\circ} \mathrm{C}$ and the supernatant was collected in 1.5 $\mathrm{ml}$ tubes. Protein concentrations were measured using the Bradford Reagent (Sigma-Aldrich) according to the manufacturer's protocol. Thirty $\mu \mathrm{g}$ of protein were then mixed with $4 \times$ Laemmli Sample Buffer (Bio-Rad) and the volume was adjusted to $25 \mu \mathrm{l}$ with lysis buffer. Subsequently, the samples were denatured at $95^{\circ} \mathrm{C}$ for $5 \mathrm{~min}$ and then separated by size on a 10\% Bis-Tris gel in $1 \times$ MOPS buffer (both Life Technologies) by applying $130 \mathrm{~V}$ for $120 \mathrm{~min}$. Subsequently, the proteins were transferred onto a nitrocellulose membrane (pore size: $0.2 \mu \mathrm{m}$ ) (Millipore) by using $1 \times$ Towbin buffer and applying $120 \mathrm{~V}$ for $120 \mathrm{~min}$. The membrane was then cut into pieces at $\sim 40$ and $\sim 80 \mathrm{kDa}$ and blocked for 1 hour using 5\% milk powder (Roth) in trisbuffered saline (TBS) (ChemCruz) supplemented with $1 \%$ Tween (Roth). The middle part of the membrane was incubated with a primary antibody against $\alpha$-tubulin (Santa Cruz, sc-32293, 1:1000) and the other parts were incubated with a primary antibody against the HA-tag (Santa Cruz, sc-7392, 1:1000), diluted in 5\% milk powder in TBS-T. After overnight incubation, the membranes were washed three time with TBS-T for 10 minutes and then incubated with HRP-(horse radish peroxidase-)linked secondary antibodies (anti-mouse anti- body, 1:5000 in 5\% milk in TBS-T (Dianova) for $1 \mathrm{~h}$. Finally, the membranes were washed again three times with TBS-T for $10 \mathrm{~min}$ and SuperSignal West Pico PLUS Chemiluminescent Substrate (ThermoFisher) was applied for $5 \mathrm{~min}$. The luminescence signal was detected using a ChemoStar detector (INTAS). The full-length western blot image is shown in Supplementary Figure S1.

\section{Computational models of AcrIIC3-LOV2 hybrids}

We used the Rosetta remodel application (58) to generate the AcrIIC3-LOV2 domain insertions based on the structures of AcrIIC3 (PDB 6J9N) and the LOV2 domain (PDB 2V0W). The N-terminus of the LOV2 structure contained three residues that were not part of our final design and thus omitted. Terminal regions of the LOV2 domain were rebuilt, including the added glycine-linkers. For rebuilding, fragment insertion with cyclic coordinate descent (59) and kinematic closure $(60,61)$ with default parameters were used. For each of the variants, 1000 decoys were generated, of which 236 passed the chain-break filter for the AcrIIC3LOV2 hybrid CN-C3G and 206 for the direct fusion CN-C3 (see below). These were subsequently clustered with a root mean square deviation threshold of $5 \AA$ into 17 clusters for $\mathrm{CN}-\mathrm{C} 3 \mathrm{G}$ and 8 clusters for the direct fusion $\mathrm{CN}-\mathrm{C} 3$.

\section{Analysis of residue contacts}

The contact map was generated on the basis of a published AcrIIC3 structure (PDB 6J9N) using PyMol 2.4 contact map visualizer (The PyMOL Molecular Graphics System, Version 2.0 Schrödinger, LLC.). Distances shorter than 7 $\AA$ were considered actual contacts. Additionally, AcrIIC3's secondary structure elements (derived from 6J9N) were plotted along the Y-axis and the tested LOV2-insertion sites as indicated in Figure 1B were highlighted in red or green.

\section{Statistical analysis}

Bars indicate means, individual data points represent individual biological replicates, i.e. independent experiments performed on different days. For the luciferase experiments, each individual data point further represents the mean of 
three technical replicates, i.e. three separate wells of a 96well plate transfected and treated in parallel. Error bars indicate the standard deviation (SD). Data analysis was performed with $\mathrm{R}$ (3.6.0).

\section{RESULTS}

To create a photo-sensitive AcrIIC3 variant, we aimed at harnessing the LOV2 domain from Avena sativa (As) phototropin-1, which is a well-characterized conformational switch (Figure 1A). The AsLOV2 domain carries two terminal helices denoted $\mathrm{J} \alpha$ and $\mathrm{A}^{\prime} \alpha$, which, in the dark state, are docked against the LOV2 protein core so that the domain's termini are in close proximity (distance $\sim 10 \AA$ ) (62). Excitation with blue light triggers the unfolding and undocking of the $\mathrm{J} \alpha$ and $\mathrm{A}^{\prime} \alpha$ helices, resulting in a massive gain of flexibility at the LOV2 termini $(63,64)$. It has previously been shown by Klaus Hahn et al. that inserting the $A s$ LOV2 domain into surface-exposed loops of enzymes can be used to disrupt their function in a light-dependent manner (65). We recently adapted this concept to engineer a light-responsive variant of the SpyCas9 inhibitor AcrIIA4 (30). By inserting the AsLOV2 domain into the most Cterminal loop of AcrIIA4 (around residue E66/Y67), we created CASANOVA (for CRISPR-Cas9 activity switching via a novel optogenetic variant of AcrIIA4), an AcrIIA4LOV2 hybrid that blocks SpyCas9 activity in the dark, but releases its function upon illumination.

When developing CASANOVA, we relied on the available structural information, which guided our selection of LOV2 insertion sites on AcrIIA4. The AcrIIC3 structure $(51,66,67)$, however, was not known at the beginning of this project. Thus, we started this work by performing a secondary structure prediction using QUARK (68) with the goal to roughly identify regions corresponding to $\alpha$-helices, $\beta$-sheets and unstructured loops, as the latter are more permissive to domain insertions.

Based on this prediction, we chose nine target regions (R1-R9) in AcrIIC3 and inserted the LOV2 domain into each of these regions. Importantly, all actual loops of AcrIIC3 were included (Figure 1B). The resulting AcrIIC3LOV2 hybrids were then screened for their ability to block NmeCas9 activity in the dark using a previously developed luciferase cleavage assay in HEK293T cells (52). In this assay, a catalytically active NmeCas 9 is targeted via a corresponding sgRNA to a firefly luciferase reporter gene (see Methods for details), thereby mediating strong reporter knockdown (Figure 1C, no Acr control). Cosupplying wild-type (wt) AcrIIC3 prevents reporter cleavage and thus results in a rescue of luciferase activity (Figure 1C, AcrIIC3). To determine a suitable experimental timing, we performed western blot analysis of protein expression following transient transfection. Both, Cas9 and the respective Acrs were detectable already $12 \mathrm{~h}$ post-transfection and reached maximum levels within $24 \mathrm{~h}$ that remained stable (Supplementary Figure S2). Hence, we decided to assess luciferase activity $48 \mathrm{~h}$ post-transfection. Remarkably, the AcrIIC3-LOV2 hybrids based on region 6 efficiently inhibited NmeCas 9 in the dark, as indicated by a potent rescue in luciferase activity comparable to that mediated by wt AcrIIC3 (Figure 1C). In contrast, the hybrids based on all other regions either showed considerably weakened inhibitory function as compared to wt AcrIIC3 (R9) or no NmeCas9 inhibition at all ( R1-5, R7-8). This suggests that LOV2 domain insertion at these sites interferes with Cas9 binding or impairs Acr folding.

Using two variants based on LOV2 insertions in R6 (Figure 1C; F59, N60), we next investigated their ability to switch activity in a light-dependent manner, using the aforementioned luciferase assay and in genome editing experiments read-out by $\mathrm{T} 7$ assay. We either exposed samples to blue light for $48 \mathrm{~h}$ (luciferase assays) or $72 \mathrm{~h}$ (T7 assays) or kept them in the dark prior to measurements. We observed potent inhibition of Cas9 in the dark and a release of Cas9 activity upon blue light irradiation (Figure 1E, F, construct \#1). To rule out that the editing was influenced by toxicity due to light treatment, we performed an MTT assay, i.e. a commonly used, colorimetric assay to determine cell viability. This showed that the blue light setup we used did not affect cell viability (Supplementary Figure S3).

Next, using the variants bearing the LOV2 domain behind AcrIIC3 residues F59 and N60 as scaffold, we also tested the effects of inserting flexible GS-linkers or a short deletion at the Acr-LOV boundaries. Most AcrIIC3-LOV2 hybrids showed strong light-dependent Cas9 inhibition, albeit the level of Cas9 activity in the light and dark condition varied considerably between the different variants (Figure 1D-F, Supplementary Figures S4). The hybrids carrying the wt LOV2 domain behind AcrIIC3 residue F59 either with or without symmetric, single-glycine linkers at the LOV2-AcrIIC3 boundaries (variants 1 and 4 in Figure 1D-F, respectively) showed particularly robust lightswitching behaviour in both assays, while blocking Cas9 activity in the dark comparably to wt AcrIIC3. We named these variants $\mathrm{CN}-\mathrm{C} 3$ (for CASASNOVA-C3) and $\mathrm{CN}$ C3G (for CASANOVA-C3 symmetric glycine linker variant), respectively.

Next, to investigate light-control when targeting NmeCas9 to different, endogenous loci as well as upon different modes of delivery, HEK293T cells were either co-transfected with plasmids or co-transduced with viral vectors based on AAV serotype 2 encoding (i) NmeCas9 and a sgRNA and (ii) $\mathrm{CN}-\mathrm{C} 3(\mathrm{G})$. Indel formation at the target locus in the presence and absence of light was then measured by targeted amplicon sequencing and further validated by $\mathrm{T} 7$ assay and TIDE sequencing (Figure 2A).

Remarkably, we observed potent, light-dependent editing at all loci tested (Figure 2B and Supplementary Figure S5). In line with previous observations in context of CASANOVA (30), the background editing in the dark was lower and the dynamic range of light-regulation slightly higher when applying AAV transduction instead of transient transfection for delivery (Supplementary Figure S5), probably due to the more homogenous expression of the different components from AAVs. Interestingly, inhibition was to some degree locus-dependent, a property which was not specific to $\mathrm{CN}-\mathrm{C} 3(\mathrm{G})$, but also observed for wt AcrIIC3 (Figure 2B and Supplementary Figure S5). When targeting the F8 locus, for instance, wt AcrIIC3 completely abolished indel formation, while for other loci, e.g. FLJ00328 considerable editing was observed also in presence of AcrIIC3. Similarly, our light-switchable CN-C3(G) system was tight 
A

Dark
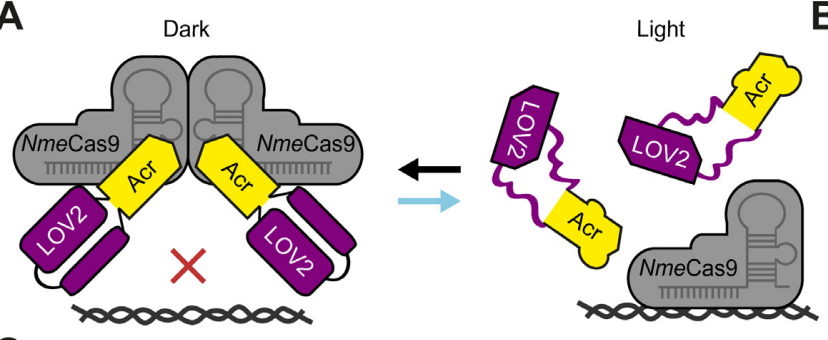

B
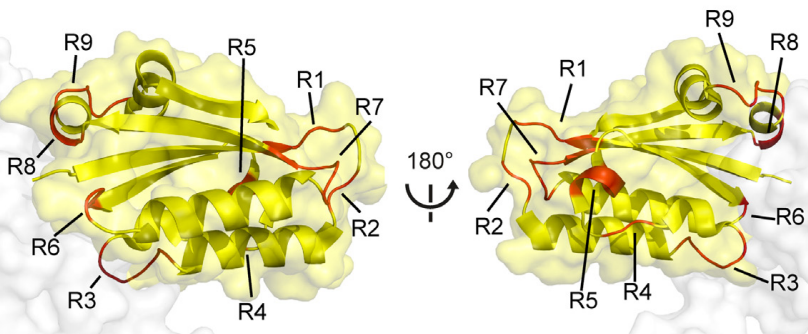

C
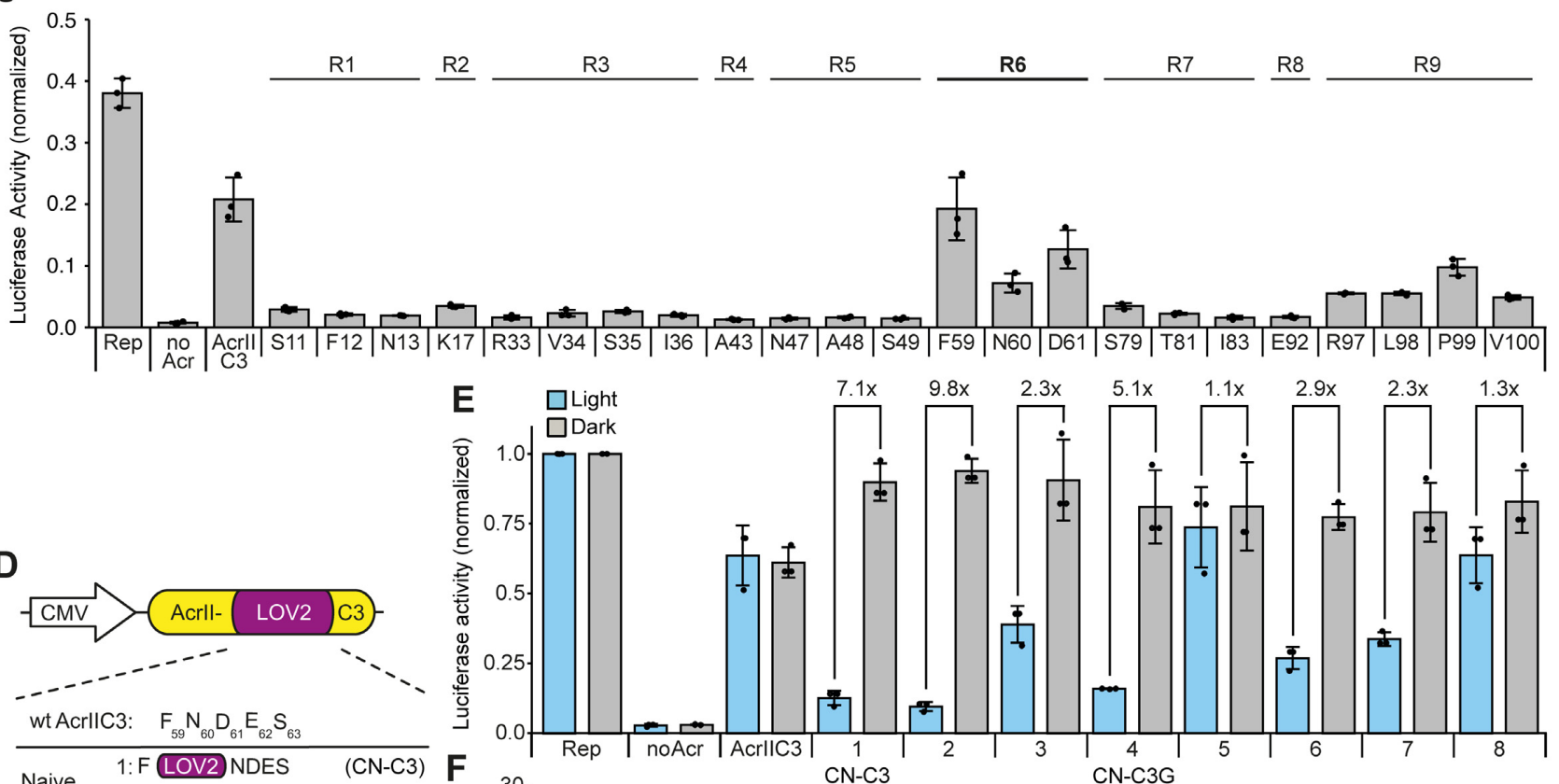

D

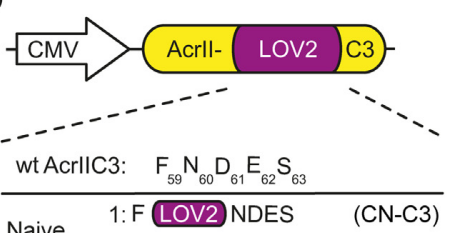

Insertion 2: FN LOV2DES

Deletion 3: FNDLOV2 S

4: FGLOV2 GNDES (CN-C3G)

5: FGSG LOV2 GSGNDES

GS-

6: FNG LOV2 GDES

7:FNGSG LOV2 GSGDES

8: FNDG LOV2S

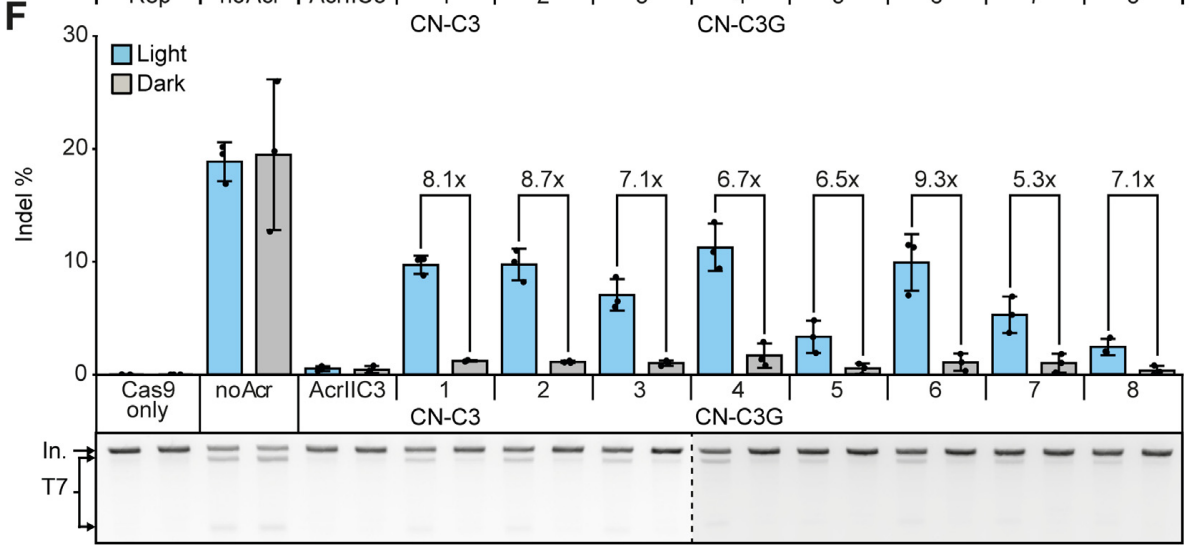

Figure 1. Engineering of CASANOVA-C3, a light-switchable anti-CRISPR protein for optogenetic control of NmeCas9. (A) Schematic of CASANOVAC3 function. (B) Structure of AcrIIC3. The nine regions chosen for LOV2 domain insertion (R1-R9) are shown in red (PDB 6J9N). (C) Luciferase reporter-based screen of AcrIIC3-LOV2 hybrids. HEK293T cells were co-transfected with vectors encoding (i) a firefly luciferase reporter, (ii) NmeCas9 and a sgRNA targeting the luciferase reporter and (iii) either wild-type AcrIIC3 (AcrIIC3) or the indicated AcrIIC3-LOV2 hybrid (S11-V100) followed by luciferase assay. The AcrIIC3 residues behind which the LOV2 domain was inserted are indicated. R1-9 correspond to the different regions in B. R, region. Rep, reporter only control. The lead region is labelled in bold. (D) Lead panel of AcrIIC3-LOV2 hybrids. Glycine-serine linkers are in green. (E) Luciferase assay screen of the AcrIIC3-LOV2 hybrids in D. Cells were transfected as in C and then exposed to blue light or kept in the dark for 48 hours, followed by luciferase assay. Rep, reporter only control. (F) HEK293T cells were co-transfected with vectors encoding (i) NmeCas9 and a sgRNA targeting the endogenous IL2RG locus and (ii) the indicated Acr variant in D. Samples were exposed to blue light or kept in the dark for $72 \mathrm{~h}$. Gene editing was assessed by T7 assay. Representative gel images are shown below the bar charts. The dotted line separates different gels. In, input. T7, T7 cleavage fragments. (C, E, F) Bars represent mean values, error bars the standard deviation and dots individual data points from $n=3$ independent experiments. 
A
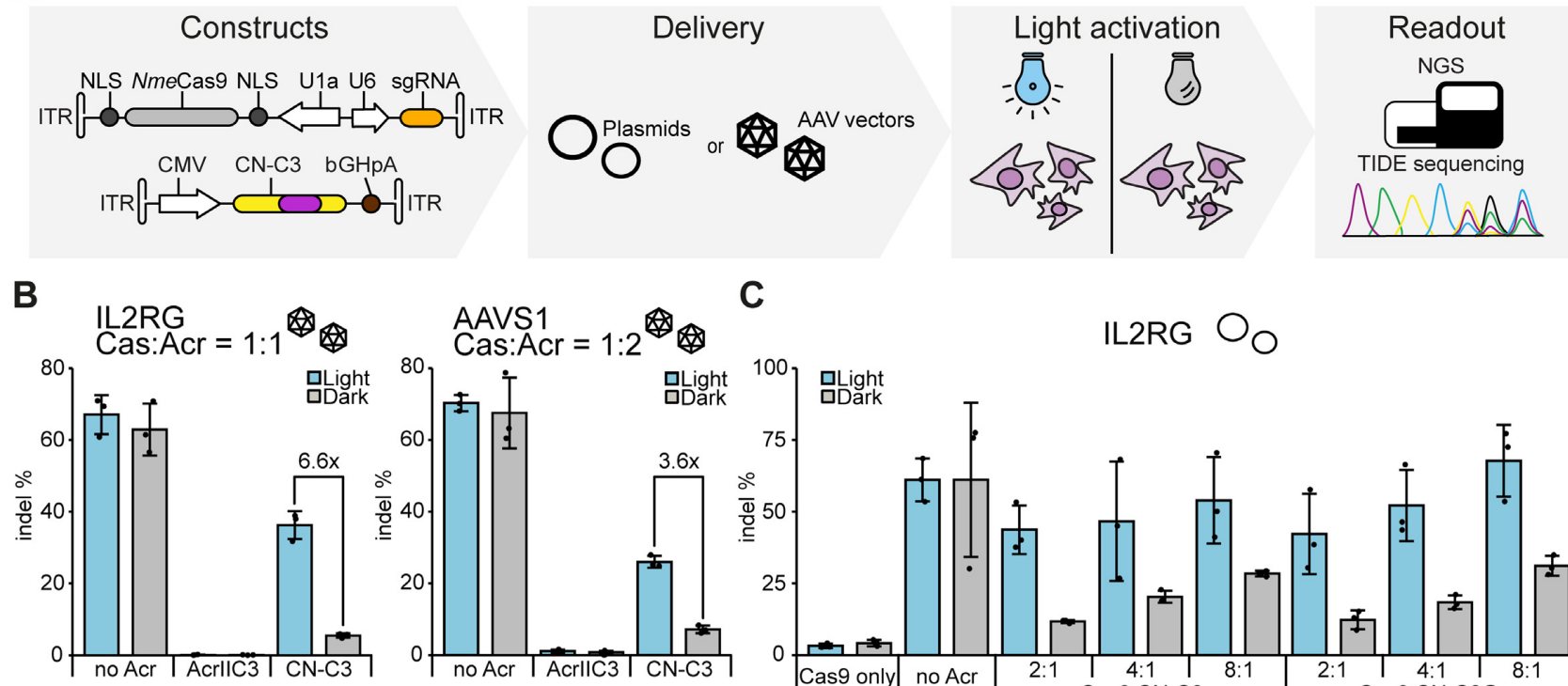

C

D
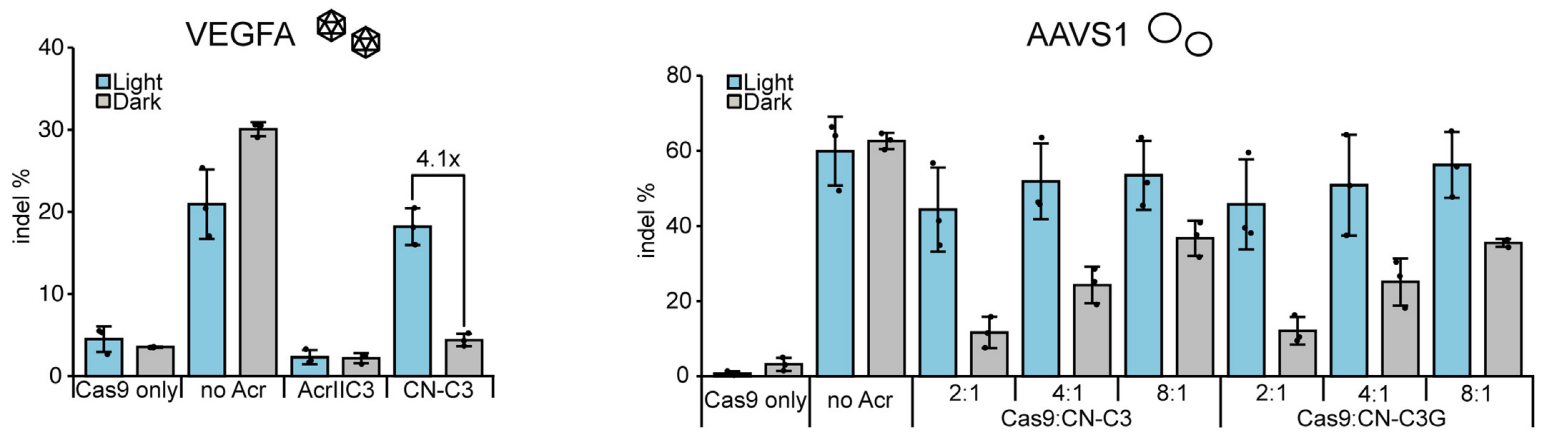

Figure 2. Light-dependent genome editing. (A) Experimental workflow. (B, C) HEK293T cells were co-transduced with AAV vectors (B) or co-transfected with plasmids (C) encoding (i) NmeCas9 and a sgRNA targeting the indicated locus and (ii) the indicated Acr variant. Cells were then irradiated with pulsed blue light or kept in the dark for 72 hours, followed by assessment of indel frequencies using NGS (B) or TIDE sequencing (C). Plasmid mass ratios used during the transfection in $\mathbf{C}$ are indicated. (D) Huh7 cells were co-transduced with AAV vectors encoding (i) NmeCas9 and a sgRNA targeting the indicated locus and (ii) the respective Acr. Seventy-two hours post-transduction, editing frequencies were determined by TIDE sequencing. (B-D) Bars represent mean values, error bars the standard deviation and dots individual data points from $\mathrm{n}=3$ independent experiments.

on some loci, while for other loci some editing also occurred in the absence of light (Supplementary Figure S5). The performance of our NmeCas9 light-switch was further dependent on the used $\mathrm{CN}-\mathrm{C} 3(\mathrm{G})$ dose. At increasing $\mathrm{CN}$ $\mathrm{C} 3(\mathrm{G})$ :NmeCas9 vector ratios, background editing in the dark was efficiently reduced, albeit at the cost of some reduction of Cas9 activity upon irradiation (Figure 2C). Interestingly, this reduction in Cas9 is accompanied by a positive effect on Cas9 specificity, i.e. off-target editing is reduced in the presence of CN-C3 (Supplementary Figure S6). The use of AAV vectors for delivery enables the application of $\mathrm{CN}-\mathrm{C} 3$ also in difficult-to-transfect cell lines as we exemplified by light-dependent genome editing in Huh7, a hepatocellular carcinoma cell line (Figure 2D). Together, these data demonstrate that our CN-C3 system is tuneable and can be used to efficiently control genome editing at various loci.

Having demonstrated optogenetic control of NmeCas9 with $\mathrm{CN}-\mathrm{C} 3(\mathrm{G})$, we finally aimed at investigating possible mechanisms of LOV2-mediated light-switching. This was particularly relevant, as we did not use specific design criteria when engineering $\mathrm{CN}-\mathrm{C} 3(\mathrm{G})$ apart from trying to confine LOV2 insertion to loops (see above). First, we performed a detailed analysis of residue contacts within AcrIIC3 to see whether the loop into which we had inserted the LOV2 domain (region 6 in Figure 1B) connects interacting secondary structures. Unlike our previously reported LOV2 insertion site underlying CASANOVA (30) (Supplementary Figure S7), as well as those of previously published LOV2-kinase hybrids $(65,69)$, the target loop within AcrIIC3 region 6 does not connect interacting secondary structures. Instead, it connects a helix and beta-sheet that stand in an angle of $\sim 40^{\circ}$ to one another (Figure 3A, B). Surprisingly, the insertion site appears to be located right at the boundary of the Cas9 binding surface (its distance to $\mathrm{HNH}$ domain is only $\sim 7 \AA$ ) and, based on the reported structures of the AcrIIC3-NmeCas9 HNH complex, would not even be considered entirely surface-exposed. In fact, the insertion site is directly flanked by residues, which mediate important contacts with the $\mathrm{HNH}$ domain 
A

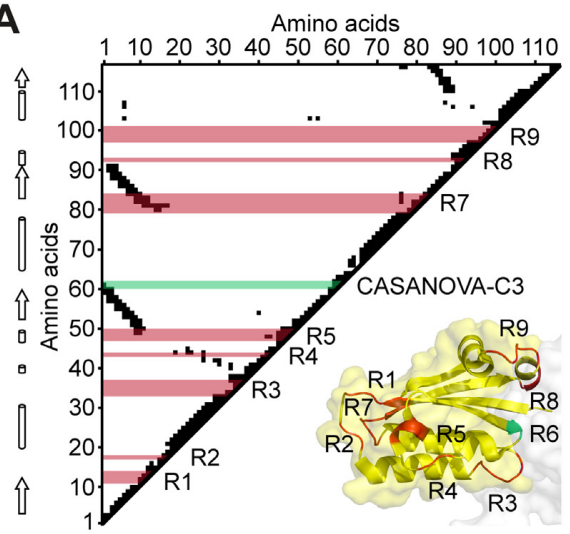

B

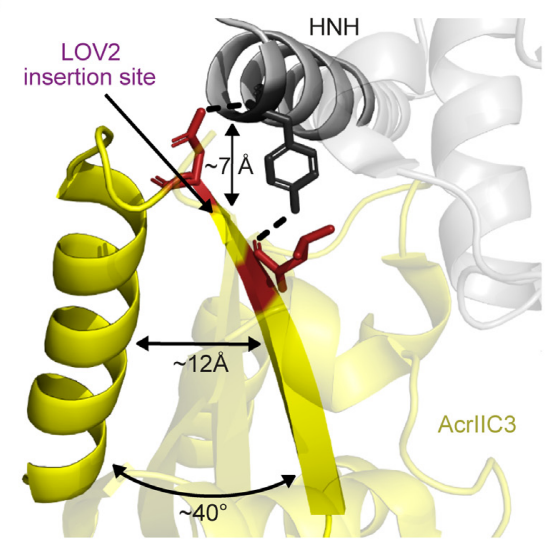

C

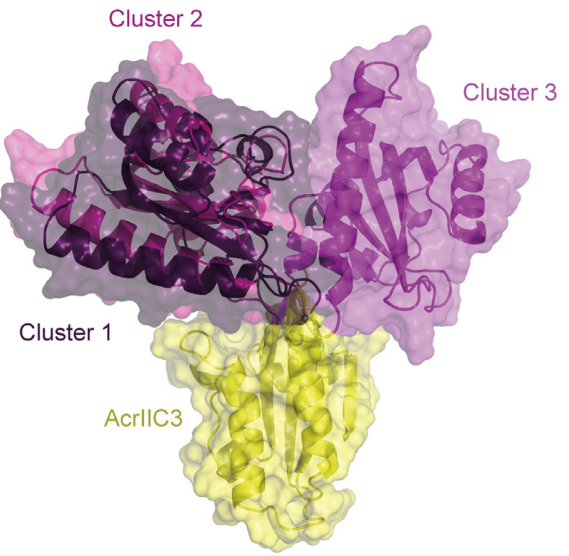

$-C_{90}^{\circ}$

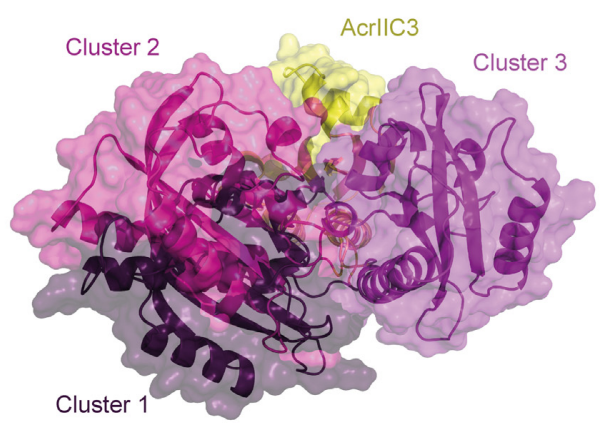

D

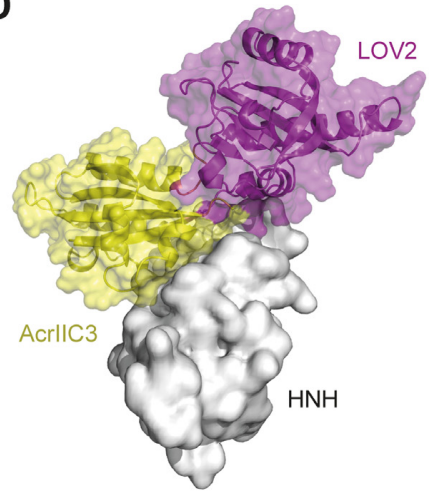

$\underbrace{90^{\circ}}_{1}$

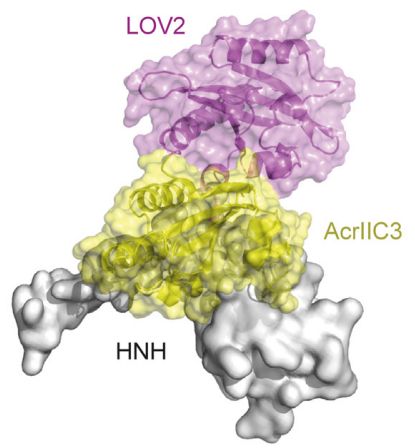

Figure 3. The LOV2 domain in CN-C3 is located in close proximity to the NmeCas9 binding surface. (A) Analysis of AcrIIC3 residue contacts. Spatially proximate AcrIIC3 residue pairs (distance $<7 \AA$ ) are indicated by black squares (triangular plot). $\alpha$-Helices and $\beta$-sheets, indicated by cylinders and arrows according to the published structure (PDB: 6J9N) are shown on the left. Regions into which the LOV2 domain was inserted into AcrIIC3 (see Figure 1B) are indicated in red and correspond to the labelled regions in the AcrIIC3 structure (lower right). The LOV2 insertion site underlying CN-C3(G) is marked in green. Numbers correspond to AcrIIC3 residues. (B) Close-up view on the identified LOV2 insertion site in context of the AcrIIC3:HNH domain complex. The approximate distance between the insertion site on AcrIIC3 and the NmeCas9 HNH domain is indicated. The angle as well as the distance between the secondary structure elements adjacent to the insertion site are shown. Residues in red mediate direct contact to the HNH domain. (C) Computational model of CN-C3 generated by domain assembly simulation. The three most populated conformational clusters of the LOV2 are shown in purple in descending order. (D) Cluster 3 does not sterically clash with the HNH-domain. PDB 6J9N, 2V0W.

(Figure 3B; AcrIIC3 L58 and N60 contacting Y540 and E539 on NmeCas9, respectively). Of note, AcrIIC3 L58 is highly conserved and absolutely critical for AcrIIC3 function (66). Finally, to investigate possible configurations of the LOV2 domain in context of $\mathrm{CN}-\mathrm{C} 3(\mathrm{G})$, we performed structural modeling. In absence of an $\mathrm{HNH}$ binding partner, we found multiple possible conformations of the LOV2 domain relative to AcrIIC3 due to considerable flexibility at the Acr-LOV2 junction sites (Figure 3C and Supplementary Figure S8A). Out of the three most populated LOV2 conformational states, however, only one (cluster 3 ) did not show considerable steric clashes with the HNH domain when the $\mathrm{CN}-\mathrm{C} 3(\mathrm{G}): \mathrm{HNH}$ complex is assembled (Figure 3D, Supplementary Figure S8B, Supplementary Figure S9A). Structural alignment of the model to the published full-length NmeCas9 bound to AcrIIC3 in 2:2 ratio (67) confirmed this result, suggesting that the position of the LOV2 domain relative to the Acr is restricted upon NmeCas9 binding (Supplementary Figure S9B). Together, these observations lead to the hypothesis that the potent light-switching behaviour of $\mathrm{CN}-\mathrm{C} 3(\mathrm{G})$ might, at least in part, result from locally induced disorder directly at the Cas 9 binding surface of AcrIIC3.

\section{DISCUSSION}

Systems to confine the activity of Cas9 in time and space are highly desired, as they improve the precision at which CRISPR-mediated genome perturbations can be made $(26,27,31)$. We had previously engineered CASANOVA, an optogenetic SpyCas9 inhibitor based on LOV2 insertion into AcrIIA4 (30). Here, we extended the CASANOVA approach to AcrIIC3, which is structurally unrelated to AcrIIA4, thereby demonstrating that LOV2 insertion into Acrs is a generalizable strategy to engineer light-switchable CRISPR inhibitors. CN-C3(G) enabled light-dependent NmeCas9 genome editing at various target loci in mammalian cells and is compatible with delivery via transient transfection or AAVs. The latter are prime vector candidates for human gene therapy applications $(70,71)$. While our investigation was limited to cell culture experiments, it 
would be interesting to explore if light-dependent Acrs can also be applied in vivo.

A particular advantage of light-switchable Acrs over photoactivatable Cas9 variants is their versatility: They are compatible with both, catalytically active Cas9 as well as dCas9-effector fusions, provided the underlying Acr impairs dCas9 DNA binding (as is the case for both, AcrIIA4 and AcrIIC3). Moreover, users can work with their established CRISPR constructs and systems such as Cas9 stable cell lines. We speculate that the future engineering of optogenetic Acrs based on broad-spectrum inhibitors such as AcrIIC1, AcrIIC1X, AcrIIA5, AcrVA1 (49,50,72-74) will further enhance their application range by enabling simultaneous regulation of multiple Cas9 orthologues. When employing $\mathrm{CN}-\mathrm{C} 3$ for light control of genome editing, it is important to consider that the editing kinetics are not only determined by the response time of $\mathrm{CN}-\mathrm{C} 3$ to light or darkness, but also affected by the protein abundance, state (Cas9 being DNA bound or free) and DNA repair kinetics. Thus, the actual response time to the light trigger will not be instantaneous, but substantial delays between Cas9 release upon illumination and the manifestation of editing are to be expected dependent on the experimental condition.

In the absence of structural information of the Acr by the time, we solely relied on sequence-based secondary structure predictions to guide our engineering efforts. Based on the structure, which is now available, we would likely not have considered the best LOV2 insertion site due to its seemingly insufficient solvent-exposure and close proximity to the Cas9 binding surface. It is important to note that only few studies have been performed in the past, in which LOV2 domain insertion sites were mapped within target proteins in an unbiased fashion (75). Moreover, most past studies using LOV2 insertion for optogenetic regulation focused on enzymes, the engineering of which might follow different design criteria as compared to controlling protein-protein interactions as we do in this work. Thus, it will be interesting to explore whether LOV2 insertion in proximity to protein binding interfaces might be a generalizable design strategy for the engineering of light-dependent protein-protein interactions. Concurrently, the unbiased mapping of LOV2 insertion sites within proteins of different origin and function might be a very interesting strategy to obtain new and powerful, yet unconventional LOV2-hybrid designs for optogenetic applications. Together, our work yielded the first tool for optogenetic control of NmeCas9-mediated genome editing and suggests a novel approach to engineer lightdependent protein-protein interactions.

\section{DATA AVAILABILITY}

The $\mathrm{CN}-\mathrm{C} 3$ and $\mathrm{CN}-\mathrm{C} 3 \mathrm{G}$ vectors are available via Addgene (Plasmid \#137191, 137192). Annotated plasmid sequences (SnapGene DNA files) are provided as Supplementary Data. Structural models of $\mathrm{CN}-\mathrm{C} 3(\mathrm{G})$ are also available as Supplementary Data. All other data is available from the corresponding authors on reasonable request. Code and data for the models of $\mathrm{CN}-\mathrm{C} 3(\mathrm{G})$ are available on GitHub (https://github.com/juzb/CASANOVA-C3).

\section{SUPPLEMENTARY DATA}

Supplementary Data are available at NAR Online.

\section{ACKNOWLEDGEMENTS}

We thank the Synthetic Biology group (BioQuant center, Heidelberg) for their support and critical feedback on the manuscript. We are thankful to Erik J. Sontheimer (University of Massachusetts Medical School, Worcester) for sharing a plasmid.

Author contributions: D.N. conceived the study. M.D.H., J.M., C.S. and D.N. designed experiments. M.D.H., S.A. and J.M. conducted experiments. M.D.H., J.M., S.A. and D.N. analysed and interpreted data. J.U.z.B and Z.H. performed computational modeling of the AcrIIC3-LOV2 hybrid structures. D.G. provided expertise on AAV vectors. R.E. and D.N. jointly directed the work with support from B.E.C.. J.M. and D.N. wrote the manuscript with support from all authors.

\section{FUNDING}

D.N. acknowledges funding via the German Research Foundation (DFG) [453202693] and the Aventis foundation; J.M. acknowledges funding via the German Academic Scholarship Foundation PhD scholarship program; M.D.H. was supported by a Helmholtz International Graduate School for Cancer Research scholarship (DKFZ, Heidelberg); B.E.C. is a grantee from the European Research Council [716058], the Swiss National Science Foundation and the Biltema Foundation; the computational simulations were performed at the CSCS - Swiss National Supercomputing Centre (grant to B.E.C.); Z.H. is supported by a grant from the Swiss National Science Foundation and by the National Center of Competence in Research in Chemical Biology; D.G. acknowledges funding from the DFG [EXC81 and TRR179, 272983813]. Funding for open access charge: Aventis Foundation and Charité, Berlin.

Conflict of interest statement. M.D.H., S.A., D.G., R.E. and D.N. are inventors on a patent application related to the engineering of conditional Acrs. D.G. is a co-founder, shareholder and chief scientific officer of the company AaviGen $\mathrm{GmbH}$.

\section{REFERENCES}

1. Mali,P., Yang,L., Esvelt,K.M., Aach,J., Guell,M., DiCarlo,J.E., Norville,J.E. and Church,G.M. (2013) RNA-guided human genome engineering via Cas9. Science, 339, 823-826.

2. Cong,L., Ran,F.A., Cox,D., Lin,S., Barretto,R., Habib,N., Hsu,P.D., Wu,X., Jiang,W., Marraffini,L.A. et al. (2013) Multiplex genome engineering using CRISPR/Cas systems. Science, 339, 819-823.

3. Jinek,M., Chylinski,K., Fonfara,I., Hauer,M., Doudna,J.A. and Charpentier,E. (2012) A programmable dual-RNA-guided DNA endonuclease in adaptive bacterial immunity. Science, 337, 816-821.

4. Makarova,K.S., Wolf,Y.I., Iranzo,J., Shmakov,S.A., Alkhnbashi,O.S., Brouns,S.J.J., Charpentier,E., Cheng,D., Haft,D.H., Horvath,P. et al. (2020) Evolutionary classification of CRISPR-Cas systems: a burst of class 2 and derived variants. Nat. Rev. Microbiol., 18, 67-83.

5. Mali,P., Aach,J., Stranges,P.B., Esvelt,K.M., Moosburner,M., Kosuri,S., Yang,L. and Church,G.M. (2013) CAS9 transcriptional activators for target specificity screening and paired nickases for cooperative genome engineering. Nat. Biotechnol., 31, 833-838. 
6. Bikard,D., Jiang,W.Y., Samai,P., Hochschild,A., Zhang,F. and Marraffini,L.A. (2013) Programmable repression and activation of bacterial gene expression using an engineered CRISPR-Cas system. Nucleic Acids Res., 41, 7429-7437.

7. Hilton,I.B., D’Ippolito,A.M., Vockley,C.M., Thakore,P.I. Crawford,G.E., Reddy,T.E. and Gersbach,C.A. (2015) Epigenome editing by a CRISPR-Cas9-based acetyltransferase activates genes from promoters and enhancers. Nat. Biotechnol., 33, 510-517.

8. Kearns,N.A., Pham,H., Tabak,B., Genga,R.M., Silverstein,N.J., Garber,M. and Maehr,R. (2015) Functional annotation of native enhancers with a Cas9-histone demethylase fusion. Nat. Methods, 12 , 401-403.

9. Liu,X.S., Wu,H., Ji,X., Stelzer,Y., Wu,X., Czauderna,S., Shu,J., Dadon,D., Young,R.A. and Jaenisch,R. (2016) Editing DNA methylation in the mammalian genome. Cell, 167, 233-247.

10. Chen,B., Gilbert,L.A., Cimini,B.A., Schnitzbauer,J., Zhang,W., Li,G.W., Park,J., Blackburn,E.H., Weissman,J.S., Qi,L.S. et al. (2013) Dynamic imaging of genomic loci in living human cells by an optimized CRISPR/Cas system. Cell, 155, 1479-1491.

11. Anton,T., Bultmann,S., Leonhardt,H. and Markaki,Y. (2014) Visualization of specific DNA sequences in living mouse embryonic stem cells with a programmable fluorescent CRISPR/Cas system. Nucleus, 5, 163-172.

12. Komor,A.C., Kim,Y.B., Packer,M.S., Zuris,J.A. and Liu,D.R. (2016) Programmable editing of a target base in genomic DNA without double-stranded DNA cleavage. Nature, 533, 420-424.

13. Gaudelli,N.M., Komor,A.C., Rees,H.A., Packer,M.S., Badran,A.H., Bryson,D.I. and Liu,D.R. (2017) Programmable base editing of A*T to $\mathrm{G}^{*} \mathrm{C}$ in genomic DNA without DNA cleavage. Nature, 551, $464-471$.

14. Hsu,P.D., Scott,D.A., Weinstein,J.A., Ran,F.A., Konermann,S., Agarwala,V., Li,Y., Fine,E.J., Wu,X., Shalem,O. et al. (2013) DNA targeting specificity of RNA-guided Cas9 nucleases. Nat. Biotechnol., 31, 827-832.

15. Pattanayak,V., Lin,S., Guilinger,J.P., Ma,E., Doudna,J.A. and Liu,D.R. (2013) High-throughput profiling of off-target DNA cleavage reveals RNA-programmed Cas9 nuclease specificity. Nat. Biotechnol., 31, 839-843.

16. Lin,Y., Cradick,T.J., Brown,M.T., Deshmukh,H., Ranjan,P., Sarode,N., Wile,B.M., Vertino,P.M., Stewart,F.J. and Bao,G. (2014) CRISPR/Cas9 systems have off-target activity with insertions or deletions between target DNA and guide RNA sequences. Nucleic Acids Res., 42, 7473-7485.

17. Lee,C.M., Cradick,T.J. and Bao,G. (2016) The Neisseria meningitidis CRISPR-Cas9 system enables specific genome editing in mammalian cells. Mol. Ther., 24, 645-654.

18. Amrani,N., Gao,X.D., Liu,P., Edraki,A., Mir,A., Ibraheim,R., Gupta,A., Sasaki,K.E., Wu,T., Donohoue,P.D. et al. (2018) NmeCas9 is an intrinsically high-fidelity genome-editing platform. Genome Biol., 19, 214.

19. Ibraheim,R., Song,C.-Q., Mir,A., Amrani,N., Xue,W. and Sontheimer,E.J. (2018) All-in-one adeno-associated virus delivery and genome editing by Neisseria meningitidis Cas9 in vivo. Genome Biol., 19, 137.

20. Esvelt,K.M., Mali,P., Braff,J.L., Moosburner,M., Yaung,S.J. and Church,G.M. (2013) Orthogonal Cas9 proteins for RNA-guided gene regulation and editing. Nat. Methods, $\mathbf{1 0}, 1116$.

21. Lee,J., Mir,A., Edraki,A., Garcia,B., Amrani,N., Lou,H.E., Gainetdinov,I., Pawluk,A., Ibraheim,R., Gao,X.D. et al. (2018) Potent Cas9 inhibition in bacterial and human cells by AcrIIC4 and AcrIIC5 Anti-CRISPR proteins. MBio, 9, e02321-18.

22. Zetsche,B., Volz,S.E. and Zhang,F. (2015) A split-Cas9 architecture for inducible genome editing and transcription modulation. Nat. Biotechnol., 33, 139-142.

23. Davis,K.M., Pattanayak,V., Thompson,D.B., Zuris,J.A. and Liu,D.R. (2015) Small molecule-triggered Cas9 protein with improved genome-editing specificity. Nat. Chem. Biol., 11, 316-318.

24. Maji,B., Moore,C.L., Zetsche,B., Volz,S.E., Zhang,F., Shoulders,M.D. and Choudhary,A. (2017) Multidimensional chemical control of CRISPR-Cas9. Nat. Chem. Biol., 13, 9-11.

25. Oakes,B.L., Nadler,D.C., Flamholz,A., Fellmann,C., Staahl,B.T., Doudna,J.A. and Savage,D.F. (2016) Profiling of engineering hotspots identifies an allosteric CRISPR-Cas9 switch. Nat. Biotechnol., 34, 646-651.
26. Zhou,X.X., Zou,X., Chung,H.K., Gao,Y., Liu,Y., Qi,L.S. and Lin,M.Z. (2018) A Single-Chain photoswitchable CRISPR-Cas9 architecture for Light-Inducible gene editing and transcription. ACS Chem. Biol., 13, 443-448.

27. Nihongaki,Y., Kawano,F., Nakajima,T. and Sato,M. (2015) Photoactivatable CRISPR-Cas9 for optogenetic genome editing. Nat. Biotechnol., 33, 755-760.

28. Nihongaki,Y., Yamamoto,S., Kawano,F., Suzuki,H. and Sato,M. (2015) CRISPR-Cas9-based photoactivatable transcription system. Chem. Biol., 22, 169-174.

29. Polstein,L.R. and Gersbach,C.A. (2015) A light-inducible CRISPR-Cas9 system for control of endogenous gene activation. Nat. Chem. Biol., 11, 198-200.

30. Bubeck,F., Hoffmann,M.D., Harteveld,Z., Aschenbrenner,S., Bietz,A., Waldhauer,M.C., Borner,K., Fakhiri,J., Schmelas,C. Dietz,L. et al. (2018) Engineered anti-CRISPR proteins for optogenetic control of CRISPR-Cas9. Nat. Methods, 15, 924-927.

31. Richter,F., Fonfara,I., Bouazza,B., Schumacher,C.H., Bratovic,M., Charpentier,E. and Moglich,A. (2016) Engineering of temperatureand light-switchable Cas9 variants. Nucleic Acids Res., 44, 10003-10014.

32. Jiang,F., Liu,J.J., Osuna,B.A., Xu,M., Berry,J.D., Rauch,B.J., Nogales,E., Bondy-Denomy,J. and Doudna,J.A. (2019) Temperature-Responsive competitive inhibition of CRISPR-Cas9. Mol. Cell, 73, 601-610.

33. Yin, Y., Yang,B. and Entwistle,S. (2019) Bioinformatics identification of anti-CRISPR loci by using homology, guilt-by-association, and CRISPR self-targeting spacer approaches. mSystems, 4, e00455-19.

34. Hynes,A.P., Rousseau,G.M., Lemay,M.L., Horvath,P., Romero,D.A., Fremaux,C. and Moineau,S. (2017) An anti-CRISPR from a virulent streptococcal phage inhibits Streptococcus pyogenes Cas9. Nat Microbiol, 2, 1374-1380.

35. Hynes,A.P., Rousseau,G.M., Agudelo,D., Goulet,A., Amigues,B., Loehr,J., Romero,D.A., Fremaux,C., Horvath,P., Doyon, Y. et al. (2018) Widespread anti-CRISPR proteins in virulent bacteriophages inhibit a range of Cas9 proteins. Nat. Commun., 9, 2919.

36. Bondy-Denomy,J., Pawluk,A., Maxwell,K.L. and Davidson,A.R. (2013) Bacteriophage genes that inactivate the CRISPR/Cas bacterial immune system. Nature, 493, 429-432.

37. Bondy-Denomy,J., Garcia,B., Strum,S., Du,M., Rollins,M.F., Hidalgo-Reyes,Y., Wiedenheft,B., Maxwell,K.L. and Davidson,A.R. (2015) Multiple mechanisms for CRISPR-Cas inhibition by anti-CRISPR proteins. Nature, 526, 136-139.

38. He,F., Bhoobalan-Chitty,Y., Van,L.B., Kjeldsen,A.L., Dedola,M., Makarova,K.S., Koonin,E.V., Brodersen,D.E. and Peng,X. (2018) Anti-CRISPR proteins encoded by archaeal lytic viruses inhibit subtype I-D immunity. Nat Microbiol, 3, 461-469.

39. Pawluk,A., Bondy-Denomy,J., Cheung,V.H., Maxwell,K.L. and Davidson,A.R. (2014) A new group of phage anti-CRISPR genes inhibits the type I-E CRISPR-Cas system of Pseudomonas aeruginosa. MBio, 5, e00896.

40. Pawluk,A., Amrani,N., Zhang,Y., Garcia,B., Hidalgo-Reyes,Y., Lee,J., Edraki,A., Shah,M., Sontheimer,E.J., Maxwell,K.L. et al. (2016) Naturally occurring off-switches for CRISPR-Cas9. Cell,167, $1829-1838$.

41. Pawluk,A., Staals,R.H., Taylor,C., Watson,B.N., Saha,S., Fineran,P.C., Maxwell,K.L. and Davidson,A.R. (2016) Inactivation of CRISPR-Cas systems by anti-CRISPR proteins in diverse bacterial species. Nat Microbiol, 1, 16085.

42. Kleinstiver,B.P., Pattanayak,V., Prew,M.S., Tsai,S.Q., Nguyen,N.T., Zheng,Z. and Joung,J.K. (2016) High-fidelity CRISPR-Cas9 nucleases with no detectable genome-wide off-target effects. Nature, 529, 490-495.

43. Forsberg,K.J., Bhatt,I.V., Schmidtke,D.T., Javanmardi,K., Dillard,K.E., Stoddard,B.L., Finkelstein,I.J., Kaiser,B.K. and Malik,H.S. (2019) Functional metagenomics-guided discovery of potent Cas9 inhibitors in the human microbiome. Elife, 8, e46540.

44. Watters,K.E., Fellmann,C., Bai,H.B., Ren,S.M. and Doudna,J.A. (2018) Systematic discovery of natural CRISPR-Cas12a inhibitors. Science, 362, 236-239.

45. Yang,H. and Patel,D.J. (2017) Inhibition mechanism of an anti-CRISPR suppressor AcrIIA4 targeting SpyCas9. Mol. Cell,67, $117-127$. 
46. Liu,L., Yin,M., Wang,M. and Wang,Y. (2019) Phage AcrIIA2 DNA mimicry: structural basis of the CRISPR and anti-CRISPR arms race. Mol. Cell, 73, 611-620.

47. Dong,D., Guo,M., Wang,S., Zhu,Y., Wang,S., Xiong,Z., Yang,J., $\mathrm{Xu}, \mathrm{Z}$. and Huang,Z. (2017) Structural basis of CRISPR-SpyCas9 inhibition by an anti-CRISPR protein. Nature, 546, 436-439.

48. Shin,J., Jiang,F., Liu,J.J., Bray,N.L., Rauch,B.J., Baik,S.H., Nogales,E., Bondy-Denomy,J., Corn,J.E. and Doudna,J.A. (2017) Disabling Cas9 by an anti-CRISPR DNA mimic. $S c i A d v, \mathbf{3}$, e1701620.

49. Knott,G.J., Thornton,B.W., Lobba,M.J., Liu,J.J., Al-Shayeb,B., Watters,K.E. and Doudna,J.A. (2019) Broad-spectrum enzymatic inhibition of CRISPR-Cas12a. Nat. Struct. Mol. Biol., 26, 315-321.

50. Harrington,L.B., Doxzen,K.W., Ma,E., Liu,J.J., Knott,G.J., Edraki,A., Garcia,B., Amrani,N., Chen,J.S., Cofsky,J.C. et al. (2017) A Broad-Spectrum inhibitor of CRISPR-Cas9. Cell, 170, 1224-1233.

51. Zhu,Y., Gao,A., Zhan,Q., Wang,Y., Feng,H., Liu,S., Gao,G., Serganov,A. and Gao,P. (2019) Diverse mechanisms of CRISPR-Cas9 inhibition by Type IIC Anti-CRISPR proteins. Mol. Cell, 74, 296-309.

52. Mathony,J., Harteveld,Z., Schmelas,C., Upmeier zu Belzen,J., Aschenbrenner,S., Hoffmann,M.D., Stengl,C., Scheck,A., Rosset,S., Grimm,D. et al. (2020) Computational design of anti-CRISPR proteins with improved inhibition potency and expanded specificity. Nat. Chem. Biol., 16, 725-730.

53. Engler,C., Kandzia,R. and Marillonnet,S. (2008) A one pot, one step, precision cloning method with high throughput capability. PLoS One, 3, e3647.

54. Hoffmann,M.D., Aschenbrenner,S., Grosse,S., Rapti,K., Domenger,C., Fakhiri,J., Mastel,M., Borner,K., Eils,R., Grimm,D. et al. (2019) Cell-specific CRISPR-Cas9 activation by microRNA-dependent expression of anti-CRISPR proteins. Nucleic Acids Res., 47, e75.

55. Hoffmann,M.D., Bubeck,F. and Niopek,D. (2020) Light-Inducible CRISPR labeling. Methods Mol. Biol., 2173, 137-150.

56. Brinkman,E.K., Chen,T., Amendola,M. and van Steensel,B. (2014) Easy quantitative assessment of genome editing by sequence trace decomposition. Nucleic Acids Res., 42, e168.

57. Pinello,L., Canver,M.C., Hoban,M.D., Orkin,S.H., Kohn,D.B., Bauer,D.E. and Yuan,G.C. (2016) Analyzing CRISPR genome-editing experiments with CRISPResso. Nat. Biotechnol., 34, 695-697.

58. Huang,P.S., Ban,Y.E., Richter,F., Andre,I., Vernon,R., Schief,W.R. and Baker,D. (2011) RosettaRemodel: a generalized framework for flexible backbone protein design. PLoS One, 6, e24109.

59. Canutescu,A.A. and Dunbrack,R.L. Jr (2003) Cyclic coordinate descent: a robotics algorithm for protein loop closure. Protein Sci., 12, 963-972.

60. Coutsias,E.A., Seok,C., Jacobson,M.P. and Dill,K.A. (2004) A kinematic view of loop closure. J. Comput. Chem., 25, 510-528.

61. Mandell,D.J., Coutsias,E.A. and Kortemme,T. (2009) Sub-angstrom accuracy in protein loop reconstruction by robotics-inspired conformational sampling. Nat. Methods, 6, 551-552.
62. Harper,S.M., Neil,L.C. and Gardner,K.H. (2003) Structural basis of a phototropin light switch. Science, 301, 1541-1544.

63. Gil,A.A., Laptenok,S.P., French,J.B., Iuliano,J.N., Lukacs,A., Hall,C.R., Sazanovich,I.V., Greetham,G.M., Bacher,A., Illarionov,B. et al. (2017) Femtosecond to millisecond dynamics of light induced allostery in the Avena sativa LOV domain. J. Phys. Chem. B, 121, 1010-1019.

64. Halavaty,A.S. and Moffat,K. (2007) N- and C-terminal flanking regions modulate light-induced signal transduction in the LOV2 domain of the blue light sensor phototropin 1 from Avena sativa. Biochemistry, 46, 14001-14009.

65. Dagliyan,O., Tarnawski,M., Chu,P.H., Shirvanyants,D., Schlichting,I., Dokholyan,N.V. and Hahn,K.M. (2016) Engineering extrinsic disorder to control protein activity in living cells. Science, 354, 1441-1444.

66. Kim,Y., Lee,S.J., Yoon,H.J., Kim,N.K., Lee,B.J. and Suh,J.Y. (2019) Anti-CRISPR AcrIIC3 discriminates between Cas9 orthologs via targeting the variable surface of the HNH nuclease domain. FEBS J., 286, 4661-4674.

67. Sun,W., Yang,J., Cheng,Z., Amrani,N., Liu,C., Wang,K., Ibraheim,R., Edraki,A., Huang,X., Wang,M. et al. (2019) Structures of Neisseria meningitidis Cas 9 complexes in catalytically poised and Anti-CRISPR-Inhibited states. Mol. Cell, 76, 938-952.

68. Xu,D. and Zhang,Y. (2012) Ab initio protein structure assembly using continuous structure fragments and optimized knowledge-based force field. Proteins, 80, 1715-1735.

69. Chu,P.H., Tsygankov,D., Berginski,M.E., Dagliyan,O., Gomez,S.M., Elston,T.C., Karginov,A.V. and Hahn,K.M. (2014) Engineered kinase activation reveals unique morphodynamic phenotypes and associated trafficking for Src family isoforms. Proc. Natl Acad. Sci. U.S.A., 111, 12420-12425.

70. Grimm,D. and Buning,H. (2017) Small but increasingly Mighty: Latest advances in AAV vector research, design, and evolution. Hum. Gene Ther., 28, 1075-1086.

71. Schmidt,F. and Grimm,D. (2015) CRISPR genome engineering and viral gene delivery: a case of mutual attraction. Biotechnol. J., 10, $258-272$.

72. Mathony,J., Harteveld,Z., Schmelas,C., Upmeier Zu Belzen,J., Aschenbrenner,S., Sun,W., Hoffmann,M.D., Stengl,C., Scheck,A., Georgeon,S. et al. (2020) Computational design of anti-CRISPR proteins with improved inhibition potency. Nat. Chem. Biol., 16, 725-730.

73. Garcia,B., Lee,J., Edraki,A., Hidalgo-Reyes,Y., Erwood,S., Mir,A., Trost,C.N., Seroussi,U., Stanley,S.Y., Cohn,R.D. et al. (2019) Anti-CRISPR AcrIIA5 potently inhibits all Cas9 homologs used for genome editing. Cell Rep., 29, 1739-1746.

74. Song,G., Zhang,F., Zhang,X., Gao,X., Zhu,X., Fan,D. and Tian,Y. (2019) AcrIIA5 inhibits a broad range of Cas9 orthologs by preventing DNA target cleavage. Cell Rep., 29, 2579-2589.

75. Reynolds,K.A., McLaughlin,R.N. and Ranganathan,R. (2011) Hot spots for allosteric regulation on protein surfaces. Cell, 147, $1564-1575$. 